\title{
Geometric Condition of Singularity of $S_{3}^{2}\left(\Delta_{M S}^{2}\right)$
}

\author{
Lijuan Chen (Corresponding author) \& Mingzhu Li \\ School of Science, Qingdao Technological University \\ Fushun Road 11, Qingdao, China \\ E-mail: chenljcool@163.com \\ Li Zhang \\ College of Information Science and Engineering, Shandong Agricultural University \\ Tai' an, China
}

\begin{abstract}
The aim of this paper is to investigate the geometric condition of singularity of $S_{3}^{2}\left(\Delta_{M S}^{2}\right)$. The algebraic of singularityof $S_{3}^{2}\left(\Delta_{M S}^{2}\right)$ is obtained in (Luo and Chen, 2005). The result of this paper will be useful to further study the geometric condition of singularity of $S_{\mu+1}^{\mu}\left(\Delta_{M S}^{\mu}\right)(\mu>3)$.
\end{abstract}

Keywords: Singularity, Spline space, Geometric condition

\section{Introduction}

The definition of multivariate spline is stated as follows(Wang, 1994): for a given partition $\Delta$ of a region $\Omega$, the linearspace

$S_{k}^{\mu}(\Delta):=\left\{s|s|_{T_{i}} \in P_{k}, s \in C^{\mu}(\Omega), \forall T_{i} \in \Delta\right\}$

is called spline space of degree $k$ with smoothness $\mu$, where $T_{i}$ is a cell of the $\Delta$ and $P_{k}$ is the polynomial space of total degree $\leq k$.

Luo \& Chen(Luo and Chen, 2005) investigated the singularity of the space $S_{\mu+1}^{\mu}\left(\Delta_{M S}^{\mu}\right)(\mu \geq 2)$ and gave out an algebraic necessary and sufficient condition to the singularity. Take $\mu=1$ for instance, i.e. Morgan-Scott triangulation. Shi(shi,1991) and Diener(Diener,1990) obtained the geometric significance of the necessary and sufficient condition of $\operatorname{dim}\left(\mathrm{S}_{2}^{1}\left(\Delta_{\mathrm{MS}}\right)\right)=7$, respectively. $\mathrm{Du}(\mathrm{Du}, 2003)$ gave another type of the necessary and sufficient condition of the singularity of $\mathrm{S}_{2}^{1}\left(\Delta_{\mathrm{MS}}\right)$ from the viewpoint of the projective geometry, that is, if the six quasi-inner edges are regarded as six points in the projective plane then they lie on a conic.

Now, we research the condition of $\mu=2$.

2. Algebraic of Singularity of $S_{3}^{2}\left(\Delta_{M S}^{2}\right)$

The singularity of the spline space $S_{3}^{2}\left(\Delta_{M S}^{2}\right)$ is investigated by Luo and Chen(Luo and Chen, 2005) using the Generation Basis method. They obtained a necessary and sufficient condition in algebraic form. $\Delta_{M S}^{2}$ is seen in Figure 1.

Denoted by 


$$
\left\{\begin{array} { l } 
{ l _ { 1 } = a _ { 1 } u + b _ { 1 } w } \\
{ l _ { 2 } = a _ { 2 } u + b _ { 2 } w } \\
{ l _ { 3 } = a _ { 3 } u + b _ { 3 } w }
\end{array} \quad \left\{\begin{array} { l } 
{ l _ { 4 } = a _ { 4 } w + b _ { 4 } v } \\
{ l _ { 5 } = a _ { 5 } w + b _ { 5 } v } \\
{ l _ { 6 } = a _ { 6 } w + b _ { 6 } v }
\end{array} \text { and } \left\{\begin{array}{l}
l_{7}=a_{7} v+b_{7} u \\
l_{8}=a_{8} v+b_{8} u \\
l_{9}=a_{9} v+b_{9} u
\end{array}\right.\right.\right.
$$

Then, the following conclusion in algebraic form is true

Theorem 1. (LuoandChen,2005) The spline space $S_{3}^{2}\left(\Delta_{M S}^{2}\right)$ is $\operatorname{singular}\left(\operatorname{dim}\left(S_{3}^{2}\left(\Delta_{M S}^{2}\right)\right)=11\right.$ ) if and only if

$$
\frac{a_{1} a_{2} a_{3}}{b_{1} b_{2} b_{3}} \cdot \frac{a_{4} a_{5} a_{6}}{b_{4} b_{5} b_{6}} \cdot \frac{a_{7} a_{8} a_{9}}{b_{7} b_{8} b_{9}}=-1
$$

Let $a, b, c$ be three distinct non-infinity lines in $P_{2}$. Denoted by the intersection points between lines $a, b, c$ and $l_{i}(i=1,2,3), l_{j}(i=4,5,6), l_{i}(i=7,8,9)$ respectively. $u=<b, c>., v=<c, a>, w=<a, b>$

Let $l_{2}^{\prime}, l_{5}^{\prime}$ and $l_{8}^{\prime}$ be

$$
l_{2}^{\prime}=b_{2} u+a_{2} w, l_{5}^{\prime}=b_{5} w+a_{5} v, l_{8}^{\prime}=b_{8} v+a_{8} u .
$$

Without loss of generality, we assume that the six points determined by intersections of $\mathrm{Aa}, \mathrm{Bb}, \mathrm{Cc}$ and intersections of $l_{2}^{\prime}, l_{5}^{\prime}, l_{8}^{\prime}$ are distinct from each other in the triangulation. Under this assumption, we shall prove the following important conclusion.

Theorem 2. The spline space $s_{3}^{2}\left(\Delta_{M S}^{2}\right)$ is singular if and only if the six points determined by intersections of $\mathrm{Aa}, \mathrm{Bb}$, $\mathrm{Cc}$ and intersections of $l_{2}^{\prime}, l_{5}^{\prime}, l_{8}^{\prime}$ lie on a conic.

Proof: Without loss of generality, we regard the lines $u, v, w$ as basic lines, and let $u=(1,0,0), w=(0,1,0), v=(0,0$, 1).

From (1), we have

$$
\begin{array}{lll}
l_{1}=\left(a_{1}, b_{1}, 0\right) & l_{4}=\left(0, a_{4}, b_{4}\right) & l_{7}=\left(b_{7}, 0, a_{7}\right) \\
l_{3}=\left(a_{3}, b_{3}, 0\right), & l_{6}=\left(0, a_{6}, b_{6}\right), \text { and } & l_{9}=\left(b_{9}, 0, a_{9}\right) \\
l_{2}^{\prime}=\left(b_{2}, a_{2}, 0\right), & l_{5}^{\prime}=\left(0, b_{5}, a_{5}\right), & l_{8}^{\prime}=\left(a_{8}, 0, b_{8}\right)
\end{array}
$$

and

$$
\begin{aligned}
& A=l_{1} \times l_{9}=\left(b_{1} a_{9},-a_{1} a_{9},-b_{1} b_{9}\right), B=l_{6} \times l_{7}=\left(a_{6} a_{7}, b_{6} b_{7}-a_{6} b_{7}\right), C=l_{3} \times l_{4}=\left(b_{3} b_{4},-a_{3} a_{4}, a_{3} a_{4}\right) \\
& a=w \times v=(1,0,0), b=u \times w=(0,0,1), c=u \times v=(0,-1,0) .
\end{aligned}
$$

So the lines $\mathrm{Aa}, \mathrm{Bb}$ and $\mathrm{Cc}$ can be expressed as follows:

$$
A a=A \times a=\left(0,-b_{1} b_{9}, a_{1} a_{9}\right), B b=B \times b=\left(b_{6} b_{7},-a_{6} a_{7}, 0\right), C c=C \times c=\left(a_{3} a_{4}, 0,-b_{3} b_{4}\right) .
$$

By direct calculations, the intersections of $\mathrm{Aa}, \mathrm{Bb}, \mathrm{Cc}$ and the intersections of $\mathrm{l}_{2}^{\prime}, \mathrm{l}_{5}^{\prime}, \mathrm{l}_{8}^{\prime}$ are formed to be

$$
\begin{gathered}
v_{1}=A a \times B b=\left(a_{1} a_{9} a_{6} a_{7}, a_{1} a_{9} b_{6} b_{7}, b_{1} b_{9} b_{6} b_{7}\right) \quad, \quad v_{2}=B b \times C c=\left(a_{6} a_{7} b_{3} b_{4}, b_{6} b_{7} b_{3} b_{4}, a_{6} a_{7} a_{3} a_{4}\right), \\
v_{3}=C c \times A a=\left(-b_{3} b_{4} b_{1} b_{9},-a_{3} a_{4} a_{1} a_{9},-a_{3} a_{4} b_{1} b_{9}\right) \quad, \quad v_{4}=l_{2}^{\prime} \times l_{5}^{\prime}=\left(a_{2} a_{5},-b_{2} a_{5}, b_{2} a_{5}\right) \\
v_{5}=l_{5}^{\prime} \times l_{8}^{\prime}=\left(b_{5} b_{8}, a_{5} a_{8},-b_{5} a_{8}\right), v_{6}=l_{8}^{\prime} \times l_{2}^{\prime}=\left(-b_{8} a_{2}, b_{8} b_{2}, a_{8} a_{2}\right)
\end{gathered}
$$

We now give the equivalent condition that $v_{1}, v_{2}, \cdots, v_{6}$ lie on a conic by Pascal's Theorem. To do this, the three intersection points of three subtense of the hexagon with vertices $v_{1}, v_{2}, \cdots, v_{6}$ are

$$
\begin{aligned}
& B_{1}=\left(v_{1} \times v_{5}\right) \times\left(v_{2} \times v_{6}\right)=\left(b_{1} b_{5} b_{6} b_{7} b_{8} b_{9}+a_{1} b_{5} a_{6} a_{7} a_{8} a_{9}\right)\left(b_{2} b_{3} b_{4} a_{6} a_{7} b_{8}+a_{2} b_{3} b_{4} b_{6} b_{7} b_{8}\right) \\
& -\left(a_{1} a_{5} a_{6} a_{7} a_{8} a_{9}-a_{1} b_{5} b_{6} b_{7} b_{8} a_{9}\right)\left(-a_{2} a_{3} a_{4} a_{6} a_{7} b_{8}-a_{2} b_{3} b_{4} a_{6} a_{7} a_{8}\right)\left(a_{1} a_{5} a_{6} a_{7} a_{8} a_{9}-\right.
\end{aligned}
$$


$\left.a_{1} b_{5} b_{6} b_{7} b_{8} a_{9}\right)\left(a_{2} b_{3} b_{4} b_{6} b_{7} a_{8}-b_{2} a_{3} a_{4} a_{6} a_{7} b_{8}\right)-\left(-a_{1} b_{5} b_{6} b_{7} a_{8} a_{9}-b_{1} a_{5} b_{6} b_{7} a_{8} b_{9}\right)$

$\left(a_{6} a_{7} b_{3} b_{4} b_{8} b_{2}+b_{6} b_{7} b_{3} b_{4} b_{8} a_{2}\right)\left(-a_{1} a_{9} b_{6} b_{7} b_{5} a_{8}-b_{1} b_{9} b_{6} b_{7} a_{5} a_{8}\right)\left(-a_{6} a_{7} a_{3} a_{4} a_{2} b_{8}\right.$

$\left.-a_{6} a_{7} b_{3} b_{4} a_{2} a_{8}\right)-\left(b_{1} b_{9} b_{6} b_{7} b_{5} b_{8}+a_{1} a_{9} a_{6} a_{7} b_{5} a_{8}\right)\left(b_{6} b_{7} b_{3} b_{4} a_{8} a_{2}-a_{6} a_{7} a_{3} a_{4} b_{2} b_{8}\right)$,

$B_{2}=\left(v_{1} \times v_{4}\right) \times\left(v_{3} \times v_{6}\right)=\left(b_{1} a_{2} a_{5} b_{6} b_{7} b_{9}-a_{1} b_{2} b_{5} a_{6} a_{7} a_{9}\right)\left(-b_{1} b_{2} b_{3} b_{4} b_{8} b_{9}-a_{1} a_{2} a_{3} a_{4} b_{8} a_{9}\right)$

$-\left(-a_{1} b_{2} a_{5} a_{6} a_{7} a_{9}-a_{1} a_{2} a_{5} b_{6} b_{7} a_{9}\right)\left(b_{1} a_{2} a_{3} a_{4} b_{8} b_{9}+b_{1} a_{2} b_{3} b_{4} a_{8} b_{9}\right)\left(-a_{1} b_{2} a_{5} a_{6} a_{7} a_{9}-\right.$

$\left.a_{1} a_{2} a_{5} b_{6} b_{7} a_{9}\right)\left(-a_{1} a_{2} a_{3} a_{4} a_{8} a_{9}+b_{1} b_{2} a_{3} a_{4} b_{8} b_{9}\right)-\left(a_{1} b_{2} b_{5} b_{6} b_{7} a_{9}+b_{1} b_{2} a_{5} b_{6} b_{7} a_{8} b_{9}\right)$

$\left(-b_{3} b_{4} b_{1} b_{9} b_{8} b_{2}-a_{3} a_{4} a_{1} a_{9} b_{8} a_{2}\right)\left(a_{1} a_{9} b_{6} b_{7} b_{2} b_{5}+b_{1} b_{9} b_{6} b_{7} b_{2} a_{5}\right)\left(a_{3} a_{4} b_{1} b_{9} b_{8} a_{2}\right.$

$\left.+b_{3} b_{4} b_{1} b_{9} a_{8} a_{2}\right)-\left(b_{1} b_{9} b_{6} b_{7} a_{2} a_{5}-a_{1} a_{9} a_{6} a_{7} b_{2} b_{5}\right)\left(-a_{3} a_{4} a_{1} a_{9} a_{8} a_{2}+a_{3} a_{4} b_{1} b_{9} b_{8} b_{2}\right)$,

$\mathrm{B}_{3}=\left(\mathrm{v}_{2} \times \mathrm{v}_{4}\right) \times\left(\mathrm{v}_{3} \times v_{5}\right)=\left(a_{6} a_{7} a_{3} a_{4} a_{2} a_{5}-a_{6} a_{7} b_{3} b_{4} b_{2} b_{5}\right)\left(-b_{3} b_{4} b_{1} b_{9} a_{5} a_{8}+a_{3} a_{4} a_{1} a_{9} b_{5} b_{8}\right)$

$-\left(-a_{6} a_{7} b_{3} b_{4} b_{2} a_{5}-b_{6} b_{7} b_{3} b_{4} a_{2} a_{5}\right)\left(-a_{3} a_{4} b_{1} b_{9} b_{5} b_{8}-b_{3} b_{4} b_{1} b_{9} b_{5} b_{8}\right)\left(-a_{6} a_{7} b_{3} b_{4} b_{2} a_{5}\right.$

$\left.-b_{6} b_{7} b_{3} b_{4} a_{2} a_{5}\right)\left(a_{3} a_{4} a_{1} a_{9} b_{5} a_{8}+a_{3} a_{4} b_{1} b_{9} a_{5} a_{7}\right)-\left(b_{6} b_{7} b_{3} b_{4} b_{2} b_{5}+a_{6} a_{7} a_{3} a_{4} b_{2} a_{5}\right)$

$\left(-b_{3} b_{4} b_{1} b_{9} a_{5} a_{8}+a_{3} a_{4} a_{1} a_{9} b_{5} b_{8}\right)\left(b_{6} b_{7} b_{3} b_{4} b_{2} b_{5}+a_{6} a_{7} a_{3} a_{4} b_{2} a_{5}\right)\left(-a_{3} a_{4} b_{1} b_{9} b_{5} b_{8}\right.$

$\left.-b_{3} b_{4} b_{1} b_{9} b_{5} a_{8}\right)-\left(a_{6} a_{7} a_{3} a_{4} a_{2} a_{5}-a_{6} a_{7} b_{4} b_{3} b_{2} b_{5}\right)\left(a_{3} a_{4} a_{1} a_{9} b_{5} b_{8}+a_{3} a_{4} b_{1} b_{9} a_{5} a_{8}\right)$

The directed area of triangle determined by $B_{1}, B_{2}$ and $B_{3}$ is

$\left(B_{1}, B_{2}, B_{3}\right)=-\left(b_{5} b_{8} b_{2}+a_{2} a_{5} a_{8}\right)^{2}\left(b_{7} b_{6} b_{2}+b_{2} a_{6} a_{7}\right)\left(b_{5} b_{1} b_{9}+a_{1} a_{5} a_{9}\right)\left(b_{3} a_{8} b_{4}+a_{3} a_{4} b_{8}\right)$

$\left(b_{1} b_{3} b_{4} b_{6} b_{7} b_{9}-a_{1} a_{3} a_{4} a_{6} a_{7} a_{9}\right)^{2}\left(b_{1} b_{2} b_{3} b_{4} b_{5} b_{6} b_{7} b_{8} b_{9}+a_{1} a_{2} a_{3} a_{4} a_{5} a_{6} a_{7} a_{8} a_{9}\right)$

Since the six points $v_{1}, v_{2}, \cdots, v_{6}$ are all distinct, we have

$-\left(b_{5} b_{8} b_{2}+a_{2} a_{5} a_{8}\right)^{2}\left(b_{7} b_{6} b_{2}+b_{2} a_{6} a_{7}\right)\left(b_{5} b_{1} b_{9}+a_{1} a_{5} a_{9}\right)\left(b_{3} b a_{8} b_{4}+a_{3} a_{4} b_{8}\right)$

$\left(b_{1} b_{3} b_{4} b_{6} b_{7} b_{9}-a_{1} a_{3} a_{4} a_{6} a_{7} a_{9}\right)^{2} \neq 0$.

Hence, it follows from Pascal's Theorem (says that $v_{1}, v_{2}, \cdots, v_{6}$ lie on a conic if an only if $\left(B_{1}, B_{2}, B_{3}\right)=0$ that the necessary and sufficient condition that $v_{1}, v_{2}, \cdots, v_{6}$ lie on a conic is

$\frac{a_{1} a_{2} a_{3}}{b_{1} b_{2} b_{3}} \cdot \frac{a_{4} a_{5} a_{6}}{b_{4} b_{5} b_{6}} \cdot \frac{a_{7} a_{8} a_{9}}{b_{7} b_{8} b_{9}}=-1$

\section{Example}

In this section, we shall give two examples to illustrate our main results distinctly. One of the conic is elliptic conic, the other is hyperbolic conic.

Example 1. Consider a given triangulation shown in Fig. 2, where

$$
\begin{aligned}
& A=(1 / 2,2,1), B=(-4,-2,1), C=(4,-2,1), a=(0,-1,1), b=(1,0,1), \\
& c=(-1,0,1), u:-y=0, v:-x-y-z=0, w: x-y-z=0, \\
& l_{1}:-4 x-y+4 / 3 z=0, l_{2}:-2 / 3 x-y+2 / 3 z=0, l_{3}:-1 / 4 x-y-z=0, \\
& l_{4}: 1 / 4 x-y-z=0, l_{5}: 2 / 3 x-y+2 / 3 z=0, l_{6}: 4 / 3 x-y+4 / 3 z=0 \\
& l_{7}: 2 x-y-2 z=0, l_{8}:-1 / 2 x-y-z=0, l_{9}:-42 / 47 x-y-42 / 47 z=0 .
\end{aligned}
$$

It can be proved that the spline space $S_{3}^{2}\left(\Delta_{M S}^{2}\right)$ of piecewise polynomial of degree 3 with smoothness 2 is singular. The corresponding $l_{7}^{\prime}, l_{8}^{\prime}, l_{9}^{\prime}$ are 
$l_{7}^{\prime}=-x-y+z=0, l_{8}^{\prime}=1 / 2 x-y-z=0, l_{9}^{\prime}=-5 / 47 x-y-5 / 47 z=0$.

In this example, the conic corresponding to Theorem 2 is

$302 x^{2}-2861 x y+15800 y^{2}-1421 x+12880 y+2624=0$,

which forms elliptic conic and is shown in Fig. 2

Example 2. The following example shows that a conic corresponding to Theorem 2 forms hyperbolic conic. Given a singular triangulation $\Delta_{M S}^{2}$ for spline space of piecewise polynomial of degree 3 with smoothness shown in Fig. 3, where

$$
\begin{aligned}
& A=(1 / 2,2,1), B=(-4,-3,1), C=(3,-4,1), a=(0,-2,1), b=(1,-1,1), \\
& \quad c=(-1,0,1), u:-1 / 2 x-y-1 / 2 z=0, v:-2 x-y-2 z=0, w: x-y-2 z=0, \\
& l_{1}:-6 x-y+5 z=0, l_{2}:-3 / 2 x-y+1 / 2 z=0, l_{3}:-2 / 3 x-y-2 z=0, \\
& l_{4}: 1 / 4 x-y-2 z=0, l_{5}: x-y+z=0, l_{6}: 4 / 3 x-y+4 / 3 z=0, \\
& l_{7}: 1 / 7 x-y-8 / 7 z=0, l_{8}: 2 x-y-2 z=0, l_{9}:-38 / 61 x-y-38 / 61 z=0
\end{aligned}
$$

The corresponding $l_{7}^{\prime}, l_{8}^{\prime}, l_{9}^{\prime}$ are

$l_{7}^{\prime}=5 / 14 x-19 / 14-y=0, l_{8}^{\prime}=-3 x-2-y=0, l_{9}^{\prime}=-229 / 122 x-229 / 122-y=0$,

and the corresponding duality figure of the triangulation is shown in Fig. 3. A hyperbolic curve passing through the six points as mentioned Theorem 2 is shown in 3 and

$\frac{1143}{2803} x^{2}+\frac{1028}{1963} y^{2}-\frac{1022}{715} x y-\frac{503}{256} x z+\frac{1161}{1112} y z+z=0$

\section{References}

P. Alfeld. (1985). On the dimension of multivariate piecewise polynomial functions, Proc. Biennial Dundee Conf. On Numerical Analysis, Pitman, London.

Diener, D. (1990). Instability in the dimension of spaces of bivariate piecewise polynomials of degree $2 r$ and smoothness order $r$, SIAM J. Numer. Anal., Vol. 2, No. 3, 543-551.

Hong Du. (2003). A geometric appraoch to $\operatorname{dim}\left(\mathrm{S}_{2}^{1}\left(\Delta_{\mathrm{MS}}\right)\right.$ ), (R.H. Wang Ed.), AMS/IP Studies in Advanced Mathematics, 67-70.

Luo Zhongxuan, Wang Renhong. (2006). Structure and Dimension of Multivariate Spline Space on Arbitrary Triangulation, Journal of Computational and Applied Mathematics, Vol. 195, Issues 1-2, 113-133.

Shi X. Q. (1991) The Singularity of Morgan-Scott Triangulation, CAGD, 8, 201-206.

Wang R. H., X. Q. Shi, Z. X. Luo, Z. X. Su. (2002). Multivariate Spline and its Applications, Kluwer Press, 2002, Academic Press, Beijing, 1994(in Chinese).

Z. X. Luo and L. J. Chen. (2005). The singularity of $S_{\mu+1}^{\mu}\left(\Delta_{M S}^{\mu}\right)$, J. Information and Computational Science, Vol. 2 No. 4,739-746.

Zhongxuan Luo. (2001). Generator bases of modules in and their Application, Acta Mathematica Sinica, 44(6), 983-994. 


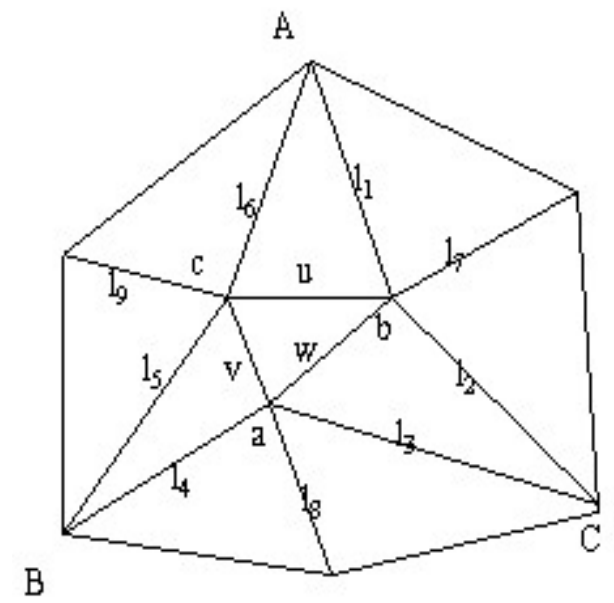

Figure 1. $\Delta^{2}{ }_{\text {MS }}$

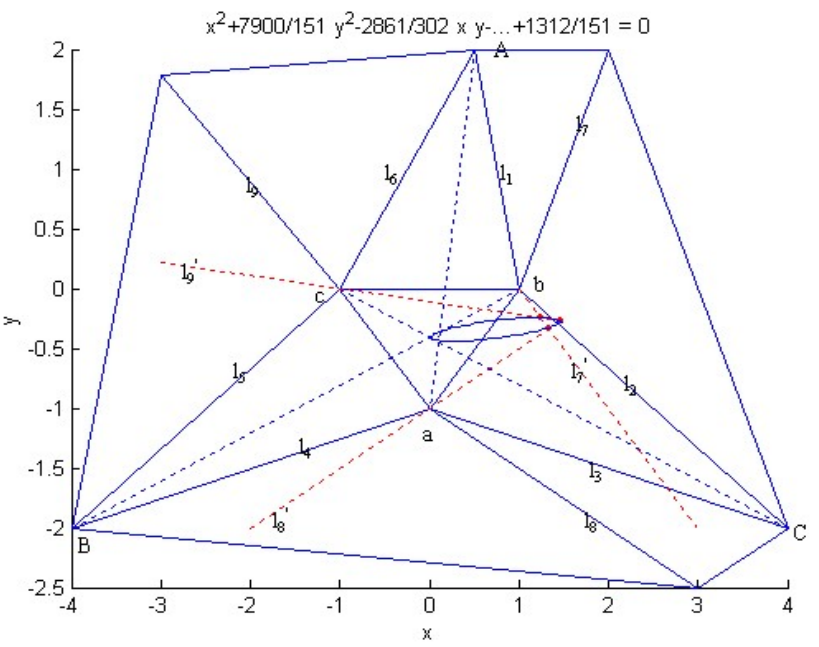

Figure 2. Example 1 


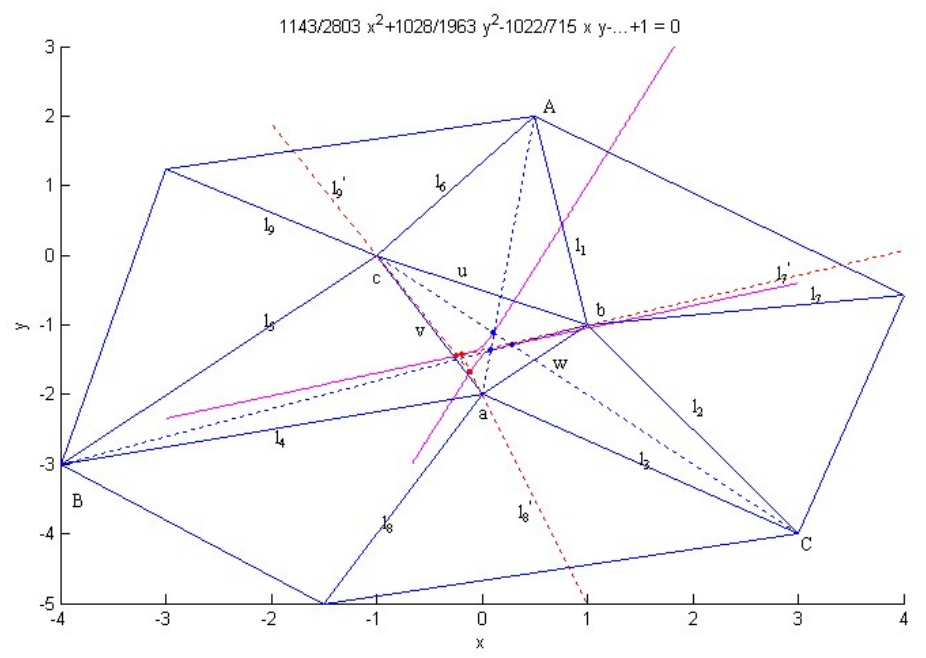

Figure 3. Example 2 